\title{
HANTAVIRUS (Bunyaviridae: Hantavirus) EN ROEDORES MURINOS DE SINCELEJO, DEPARTAMENTO DE SUCRE
}

\section{HANTAVIRUS (Bunyaviridae: Hantavirus) IN MURINE RODENTS FROM SINCELEJO, DEPARTMENT OF SUCRE}
BLANCO, T. PEDRO ${ }^{1}$ MSc., CORRALES, A. HOMER ${ }^{2^{*}}$ Biólogo, CASTELLAR, M. ANAíS ${ }^{2}$ Bióloga.

${ }^{1}$ Docente, Facultad de Educación y Ciencias, Universidad de Sucre, Colombia. Grupo de Investigaciones Biomédicas. ${ }^{2}$ Biólogos con énfasis en Biotecnología, Universidad de Sucre. Grupo de Investigaciones Biomédicas.

*Correspondencia: homerdejesuscorrales@gmail.com

\section{Resumen}

Recibido: 14-11-2012; Aceptado: 29-07-2013.

Los hantavirus, asociados a roedores de la subfamilia Sigmodontinae, son patógenosemergentes causantes de la zoonosis Síndrome Cardiopulmonar por Hantavirus (SCPH) en las Américas. Hasta la fecha, en Colombia no se han confirmado casos de la enfermedad; sin embargo, se ha demostrado la evidencia serológica de exposición a hantavirus en roedores y humanos de dos departamentos de la RegiónCaribe, y la detección molecular de genoma viral en muestras de tejido de roedores en Antioquia. El objetivo de esta investigación fue determinar la frecuencia de anticuerpos específicos contra hantavirus en roedores de la ciudad de Sincelejo, departamento de Sucre. Los muestreos de los animales fueron realizados con trampas Sherman, desde marzo hasta julio de 2009. Los anticuerpos IgG específicos contra el Virus Sin Nombre (VSN) fueron detectados en muestras de plasma medianteensayo de inmunoabsorción ligado aenzimas (ELISA indirecto). Secapturaron 61 roedores distribuidos en tres especies, de las cuales Mus musculusfue la más abundante (91.8\%). Del total de animales analizados, un único ejemplar, clasificado dentrode la especie Rattusrattus (seroprevalencia de 1.64\%), resultó positivo a hantavirus. Los hallazgos evidenciaron seropositividad a hantavirus en roedores de la subfamilia Murinae en Colombia. La detección de anticuerpos contra el virus refuerza la hipótesis que sugiere la circulación de al menos un hantavirus en roedores del norte colombiano.

Palabras clave: hantavirus, roedores, seroprevalencia, Sincelejo.

\section{Abstract}

The hantaviruses associated to the Sigmodontinae rodents, are emerging pathogens that cause the Cardiopulmonary Syndrome by Hantavirus (HCS) in the Americas. So far, Colombia has not reported confirmed cases of the disease; however, evidence has shown serological exposure to hantavirusin rodents and humans from two departments in the Caribbean Region, and 
molecular detection of viral genome in tissue samples of rodents in Antioquia. The aim of this research was to determinate the frequency of hantavirus specific antibodies in rodents from Sincelejo City. Animal samplings were performed with Sherman traps from March to July 2009. IgG antibodies against Sin Nombre Virus were detected in plasma by enzyme-linked immunosorbent assay(indirect ELISA). Sixty-one rodents were captured and identified as three speciesthree species, Musmusculus was the most abundant(91.8\%). From the total of analyzed animals, only one exemplar, belonging to Rattusrattus specie (seroprevalence 1.64\%), was positive to hantavirus. Serologic findings evidenced hantavirus infection among rodents of subfamily Murinae in Colombia. The presence of antibodies against the virus suggests that at least one hantavirus is circulating in rodents from North of Colombia.

Key words: hantavirus, rodents, seroprevalence, Sincelejo.

\section{Introducción}

Los hantavirus son un grupo de virus emergentes pertenecientes al género Hantavirus, familia Bunyaviridae(HART y BENNETT, 1999; KLEIN y CALISHER, 2007), éstos patógenos zoonóticos de roedores producen dos entidades clínicas en humanos: Fiebre Hemorrágica con Síndrome Renal (FHSR) en Eurasia y Síndrome Cardiopulmonar por Hantavirus (SCPH) en América (GONZÁLEZ et al., 2002).

El SCPH fue confirmado por primera vez en Estados Unidos, en el año 1993, ocasionado por un hantavirus denominado Virus Sin Nombre (VSN) (NICHOL et al., 1993); desde entonces, se han identificado más de 20 virus que pueden causar este síndrome en Centro y Sur América, asociados a roedores de la subfamilia Sigmodontinae (PINI et al., 2004).Actualmente, más de 2.000 casos de SCPH han sido notificados en el continente (OPS, 2004; HJELLE y TORRES-PÉREZ, 2010); aunque se trata de una enfermedad de incidencia baja, se le considera una amenaza para la salud pública de la región, porque no existe tratamiento específico para ella y por su alta tasa de mortalidad (40$50 \%$ ) (HJELLE y TORRES-PÉREZ, 2010). Esto ha despertado preocupaciónen las comunidades afectadas y el interés de las autoridades de salud pública para estudiar y prevenir esta infección (ENRIA y LEVIS, 2004).

En Colombia, no se han confirmado casos clínicos de SCPH hasta la fecha; sin embargo, se tiene evidencia serológica de contacto con hantavirus en humanos de áreas rurales de los departamentos de Córdoba y Sucre (MÁTTAR y PARRA, 2004) y en una población de roedores de los departamentos de Córdoba (ALEMÁN et al., 2006) y Sucre (BLANCO et al., 2009). En el departamento de Antioquia se detectó genoma viral de un hantavirus relacionado estrechamente con el virus Calabazo en roedores silvestres de la especie Zygodontomys brevicauda (LONDOÑO et al., 2011), previamente encontrada en Panamá. Pese a lo anterior, aún se desconocen las especies de roedores que podrían albergar el virus y las variantes del virus que circulan en las zonas urbanas del Caribe colombiano. 
El objetivo de esta investigación fue determinar la seroprevalencia de infección por hantavirus en especies de roedores que habitan áreas urbanas de la ciudad de Sincelejo, con el fin de aportar información sobre la eco-epidemiología de las hantavirosis en el departamento de Sucre.

\section{Materiales y métodos}

Área de estudio:El estudio fue realizado en la ciudad de Sincelejo, capital del departamento de Sucre (Fig. 1), ubicada en la subregión Montes de María, entre las coordenadas geográficas 9 $9^{\circ} 19^{\prime} 18^{\prime \prime} \mathrm{N}-7^{\circ} 24^{\prime} 58^{\prime \prime} \mathrm{O}, 9^{\circ} 16^{\prime} 02^{\prime \prime} \mathrm{N}$ -

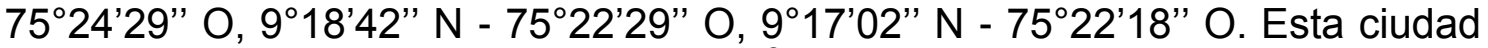
tiene una área aproximada de $21.5 \mathrm{Km}^{2}$, temperatura media de $27^{\circ} \mathrm{C}$, y una altura comprendida entre 196 y $214 \mathrm{msnm}$. Se le reconoce como una zona de bosque seco-Tropical, caracterizada por pequeñas y sucesivas colinas que incorporan múltiples caños o arroyos estacionales, rodeada de amplias zonas de pastizales para ganadería (DE LA OSSA-LACAYO y DE LA OSSA, 2011).
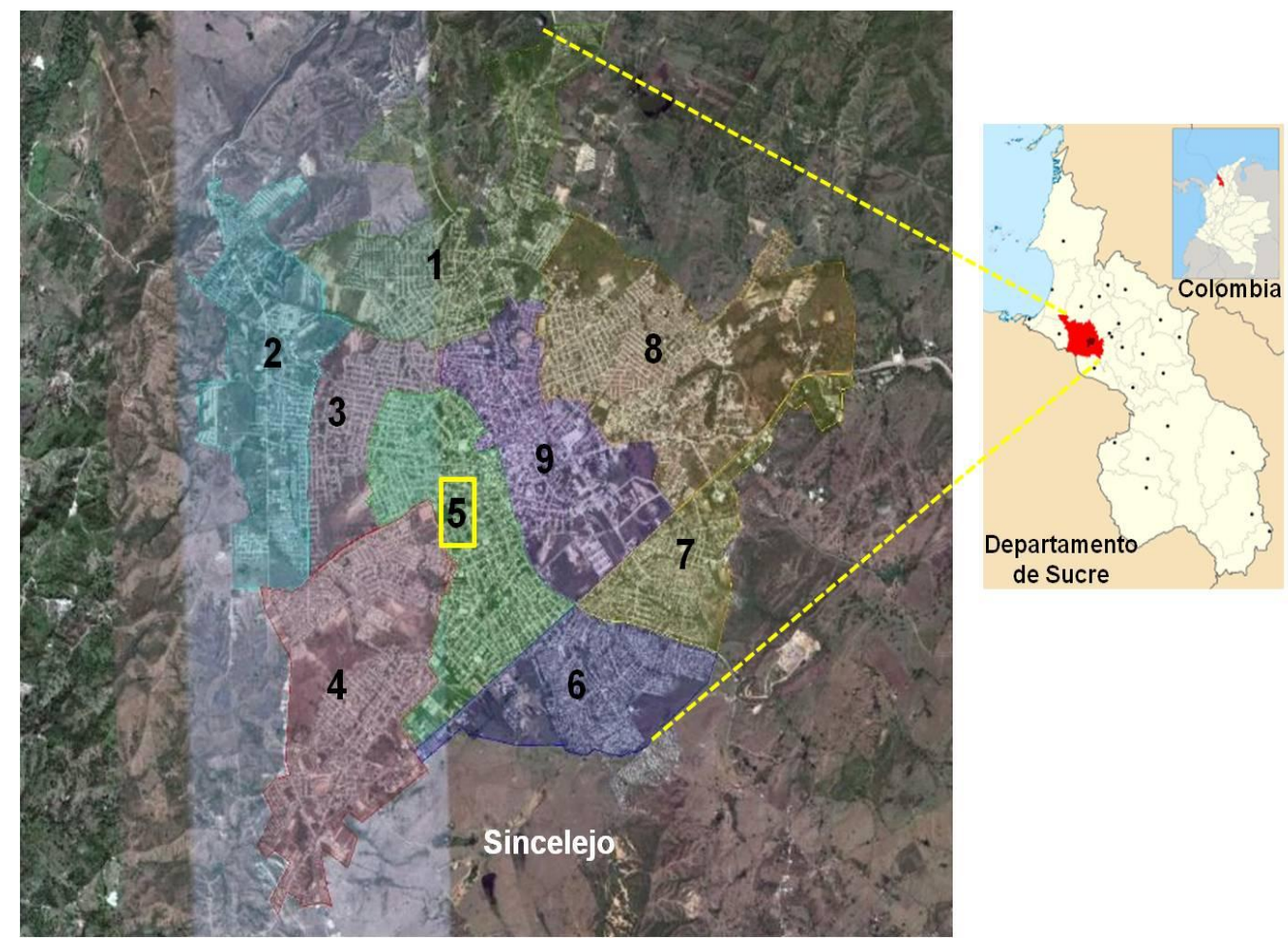

Figura 1. Distribución de las comunas del municipio de Sincelejo, departamento de Sucre (Fuente: Alcaldía de Sincelejo, 2012; GOOGLE EARTH, versión 5.0, 2012)

Trabajo de campo:Los muestreos fueron realizados por el método de remoción, siguiendo los procedimientos estandarizados para el trampeo de pequeños mamíferos (MILLS et al., 1995). Los roedores fueron capturados entre marzo y julio de 2009, mediante trampas de encierro tipo Sherman (8x9x23cm; Sherman Traps, Inc., Tallahassee, FL), las cuales fueron colocadas desde las 16:00 hasta las 06:00 horas por una noche o dos noches consecutivas y cebadas con maní o avena humedecida con vainilla. La instalación de las trampas se realizó en el intradomicilio de viviendas seleccionadas al azar, 
localizadas en nueve comunas del área urbana del municipio de Sincelejo. La autorización fue otorgada por la Corporación Autónoma Regional de Sucre (CARSUCRE), agencia de protección ambiental que rige en esta región del departamento.

Los especímenes capturados fueron transportados al laboratorio de Investigaciones Biomédicas de la Universidad de Sucre, donde se llevó a cabo su disección de acuerdo alos protocolos de bioseguridad sugeridos por el CDC (MILLS et al., 1995). Cada roedor fue anestesiado con Isoflurano y sacrificado por dislocación cervical. Los procedimientos de manejo y disección de los animales fueron aprobados por el Comité Institucional de Ética de la Universidad de Sucre.

Identificación de especímenes:se emplearon parámetros morfométricos, el cráneo y la piel de cada animal procesado para la identificación de las especies mediante claves para roedores del Nuevo Mundo (NOWAK, 1999; EMMONS y FEER, 1997).

Análisis serológico: De cada roedor procesado, fue colectada una muestra de sangre mediante punción cardiaca, centrifugada a 2500 rpm para la separación del plasma y almacenada a $-20^{\circ} \mathrm{C}$ hasta su análisis. La detección de anticuerpos lgG específicos contra el VSN fue realizada mediante la técnica de ELISA indirecto descrita previamente (SCHOUNTZ et al., 2007). El antígeno recombinante de la nucleocápside del VSN y el suero control positivo fueron donados por el Dr. C. Calisher (College of Veterinary Medicine \&Biomedical Sciences, Colorado StateUniversity). Las densidades ópticas (DO) fueron medidas a $415 \mathrm{~nm}$ en un lector de ELISA modelo 680 Bio- Rad; la densidad óptica de cada muestra fue expresada como la diferencia entre la DO de la muestra y la DO del blanco reactivo. Se asumió como punto de corte una DO de 0.250 (SCHOUNTZ et al., 2007), por lo cual, se consideraron positivos aquellos plasmas con valores por encima de este punto.

Análisis de la información:La información fue recolectada mediante un formulario estandarizado e incorporada a una base de datos. Los resultados obtenidos fueron organizados sistemáticamente mediante la estadística descriptiva. Para su análisis fueron usadas las herramientas de análisis de datos de la hoja de cálculo de Excel 2007.

\section{Resultados}

En total se capturaron 61 roedores, los cuales fueron clasificados en tres especies: 56 Mus musculus (91.8\%), 4 Rattus rattus (6,6\%) y 1 Rattus norvergicus (1,6\%); taxones pertenecientes a la familia Muridae, subfamilia Murinae (Tabla 1). 
Tabla 1. Reactividad a VSN en suero de roedores capturados en el municipio de Sincelejo, Sucre. IS: individuos seropositivos; IC: individuos capturados; \%S: porcentaje de seroprevalencia

\begin{tabular}{cccc}
\hline Familia & Subfamilia & Especie & IS/ IC (\%S) \\
\hline \multirow{3}{*}{ Muridae } & Murinae & Mus musculus & $0 / 56(0,00)$ \\
& Rattus rattus & $1 / 4(25,00)$ \\
& Rattus norvergicus & $0 / 1(0,00)$ \\
\hline \multicolumn{3}{c}{ Total } & $\mathbf{1 / 6 1}(\mathbf{1 , 6 4 )}$ \\
\hline
\end{tabular}

La prevalencia de anticuerpos específicos contra hantavirus en el total de roedores capturados fue del 1,64\% (1/61) (Fig. 2). El único individuo seropositivo correspondió a un machoen etapa juvenil de la especie Rattus rattus, capturado en la comuna 5 (sector 34 ) (Tabla 2).

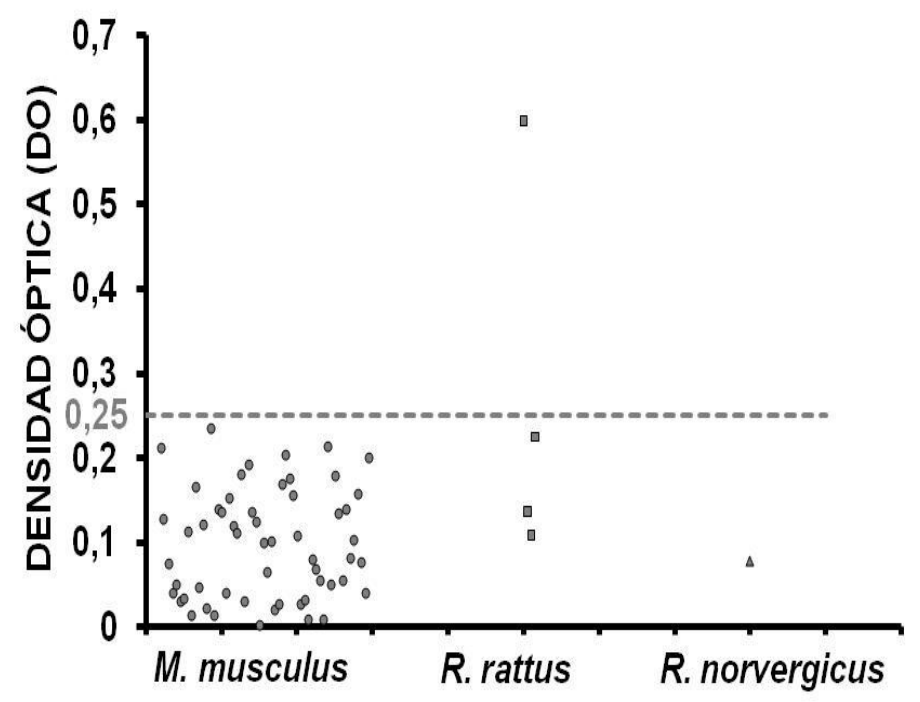

Figura 2. Valores de absorbancia a $415 \mathrm{~nm}$ detectados en las muestras de plasma de roedores analizados contra VSN. La línea discontinua representa el punto de corte

(DO: 0.25)

Tabla 2. Distribución de roedores seropositivos a hantavirus de acuerdo al sexo y etapa reproductiva. IS: individuos seropositivos; IC: individuos capturados; \%S: porcentaje de seroprevalencia

\begin{tabular}{lccccccc}
\hline \multirow{2}{*}{ Especie } & \multirow{2}{*}{ Área de muestreo } & \multicolumn{3}{c}{$\begin{array}{c}\text { Sexo } \\
\text { [IS/ IC (\%S)] }\end{array}$} & \multicolumn{2}{c}{$\begin{array}{c}\text { Etapa reproductiva } \\
\text { [IS/ IC (\%S)] }\end{array}$} \\
\hline \multirow{3}{*}{ R. rattus } & Comuna 5, Sector 34 & Macho & Hembra & Total & Juvenil & Adulto & Total \\
\cline { 3 - 8 } & & $1 / 3$ & $0 / 1$ & $1 / 4$ & $1 / 2$ & $0 / 2$ & $1 / 4$ \\
& & $(33,33)$ & $(0,00)$ & $(25,00)$ & $(50,00)$ & $(0,00)$ & $(25,00)$ \\
\hline
\end{tabular}

\section{Discusión}

Los resultados obtenidos en esta investigación constituyen el primer aporte encaminado a estudiar la circulación de hantavirus en comunidades de roedores de la ciudad de Sincelejo y el segundo en el departamento de Sucre 
(BLANCO et al., 2009). Por lo tanto, representan una herramienta valiosa en la necesidad de identificar los reservorios de estos agentes infecciosos y el análisis eco-epidemiológico de la aparición de posibles brotes en el país.

La seroprevalencia a hantavirus $(1,64 \%)$ encontrada en roedores del municipio de Sincelejo es similar a tasas de seropositividad registradas en la Octava Región de Chile (1,66\%) (ORTIZ et al., 2004) y en el departamento de Córdoba en Colombia (2.1\%) (ALEMÁN et al., 2006). Sin embargo, difiere de los porcentajes informados en algunas zonas de los Estados Unidos como New York (8,6\%) (WILSON et al., 1995) y Florida (8,2\%) (GLASS et al., 1998), y del único registro serológico previo en roedores de Sucre $(8,4 \%)$ (BLANCO et al., 2009).Estudios retrospectivos han demostrado que los hantavirus han circulado en América con anterioridad a la detección de los casos clínicos, como ocurrió en Chile con el hallazgo por serología de casos de infección por hantavirus antes de la primera epidemia de SCPH en 1995 (BARO et al., 1999; NAVARRETE et al., 2000). Por lo anterior, la detección de especies potencialmente portadoras de hantavirus, aún en ausencia de casos notificados de la enfermedad, señalan la existencia de riesgos potenciales para las poblaciones humanas en el departamento de Sucre y requieren la intensificación de medidas de vigilancia epidemiológica y prevención.

La notable diferencia en la seroprevalencia general hallada en las poblaciones de roedores de Sincelejo y San Marcos (Sucre) podría atribuirse al fenómeno de concentración focal de roedores seropositivos que pudo presentarse en San Marcos, hipótesis basada en los múltiples roedores con evidencia de anticuerpos capturados en la misma línea de trampeo (BLANCO et al., 2009). Lainfección focal por hantavirus ha sido documentada por otros autores en Estados Unidos (GLASS et al., 1998) y Argentina (PINI et al., 2003).

La presencia de anticuerpos contra hantavirus en la especie $R$. rattus representa la segunda evidencia serológica de infección por hantavirus en roedores murinos en Colombia. BLANCO et al. (2009) informaron una seroprevalencia de 2,1\% para esta misma especie en el municipio de San Marcos, departamento de Sucre. Si bien la transmisión de los hantavirus se considera de alto riesgo para los habitantes rurales por la mayor probabilidad de exposición a los roedores silvestres (SCHMALJOHN y HJELLE, 1997), estos resultados sugieren que la especie Rattus rattus estaría actuando como posible reservorio de un hantavirus circulante en el área urbana de Sincelejo. Antecedentes epidemiológicos ya habían evidenciado la infección por hantavirus en ratas urbanas del continente americano, en un estudio realizado en Brasil en 1982 se registró la presencia de anticuerpos contra el virus Seoul en Rattus rattus (LEE et al., 1982). Lo anterior sugierela posible presencia de un ciclo urbano para hantavirus en ratas domésticas de Sincelejo.

La evidencia de infección por hantavirus en humanos y roedores procedentes de dos departamentos de la Región Caribe de Colombia (MÁTTAR y PARRA, 2004; ALEMÁN et al., 2006; BLANCO et al., 2009), sumada a nuestros resultados, sugieren que la enfermedad se puede estar presentando en Sucre, 
con manifestaciones leves y subclínicas compatibles con síndromes febriles de otras causas, debido a la circulación de un hantavirus menos virulento, como se ha propuesto para otros países de Suramérica (PINI et al., 2003). Sin embargo, la falta de vigilancia epidemiológica y búsqueda activa de estos patógenos en humanos impiden la confirmación de esta hipótesis hasta ahora.

Se considera que en Colombia y especialmente en las áreas donde se ha encontrado evidencia de exposición a hantavirus como los departamentos de Sucre y Córdoba, se requieren estudios virológicos que permitan conocer los hantavirus circulantes y su relación con otros hantavirus patógenos para el hombre. Esta información permitirá mejorar el diagnóstico diferencial de los síndromes febriles sin foco evidente y planear estrategias de intervención, como educación a grupos poblacionales en riesgo, y otras medidas importantes de salud pública para prevenir la aparición de futuros brotes de esta enfermedad emergente en el país.

\section{Conclusión}

La presencia de anticuerpos contra VSN en $R$. rattus del área urbana del municipio de Sincelejo indica que al menos un hantavirus estaría circulando en estazona y señala la existencia de riesgos de transmisión para la población humana; así mismo, denota la necesidad de implementar medidas de vigilancia ecoepidemiológica con el fin de conocer la verdadera patogenicidad de este agente infeccioso.

Agradecimientos: Agradecemos al zoólogo Vladimir Rojas por el entrenamiento en el trabajo de campo y su asesoría en la determinación taxonómica de roedores, y al Dr. Charles Calisher por la donación del antígeno y el suero usado como control positivo. Esta investigación fue financiada con recursos aportados por el Departamento Administrativo de Ciencia Tecnología e Innovación -COLCIENCIAS (Código 112934419059, Contrato RC № 326 2006) y la Universidad de Sucre.

\section{Referencias}

ALEMÁN, A.; IGUARÁN, H.; PUERTA, H.; CANTILLO, C.; MILLS, J.; ARIZ, W.; MÁTTAR, S. 2006. Primera Evidencia Serológica de Infección por Hantavirus en Roedores, en Colombia. Rev Salud Pública 8:1-12.

BARO, M.; VERGARA, J.; NAVARRETE, M. 1999. Hantavirus in Chile: review and cases analysis since 1975. Rev Med Chil. 127:1513-1523.

BLANCO, P.; CASTELLAR, A.; ÁlVAREZ, L.; ARROYO, S.; CORRALES, H.; PÉREZ, J. 2009. Evidencia serológica de infección por hantavirus (Bunyaviridae: Hantavirus) en roedores del municipio de San marcos, Sucre. Biomédica 29: 276-277 
DE LA OSSA-LACAYO, A.; DE LA OSSA, V.J. 2011. Abundancia relativa de rapaces diurnas en la ciudad de Sincelejo, Sucre, Colombia. Rev. Colombiana cienc Anim. 3: 253-261.

EMMONS, L.H.; FEER, F. 1997. Neotropical rainforest mammals: a field guide. Second Edition. The University of Chicago Press. Chicago, Illinois, EEUU.

ENRIA, D.; LEVIS, S. 2004. Zoonosis virales emergentes: las infecciones por hantavirus. Rev sci tech Offinf Epiz. 23:595-611.

GLASS, G.E.; LIVINGSTONE, W.; MILLS, J.N.; HLADY, W.G.; FINE, J.B.; BIGGLER, W.; COKE, T.; FRAZIER, D.; ATHERLEY, S.; ROLLIN, P.E.; KSIAZEK, T.G.; PETERS, C.J.; CHILDS, J.E. 1998. Black Creek Canal virus infection in Sigmodon hispidus in southern Florida. Am J Trop Med Hyg. 5:699703.

GONZÁLEZ, M.; EDELSTEIN, A.; MIGUEL, S.; MARTÍNEZ, V.; CORTEZ, J.; CACACE, M.; JURGELENAS, G.; ESTANI, S.; PADULA, P. 2002. Andes virus associated with hantavirus pulmonary syndrome in northern Argentina and determination of the precise site of infection. Am J Trop Med Hyg. 66:713-720.

GOOGLE EARTH, Version 5.0. Google Inc. Mountain View, California, USA. Disponible en: Google Earth. Consultado en marzo de 2012.

HART, C.; BENNETT, M. 1999. Hantavirus infections: epidemiology and pathogenesis. Micro Infect. 1:1229-1237.

HJELLE, B.; TORRES-PÉREZ, F. 2010. Hantaviruses in the Americas and Their Role as Emerging Pathogens. Viruses 2:2559-2586.

KLEIN, S.; CALISHER. C. 2007. Emergence and persistence of hantaviruses. Curr Top Microbiol Immunol. 317:217-252.

LEE, H.; BAEK, L.; JOHNSON, K. 1982. Isolation of Hanta an virus, the etiologic agent of Korean hemorrhagic fever, from wild urban rats.J Infect Dis. 146:638-644.

LONDOÑO, A.F.; DÍAZ, F.J.; AGUDELO-FLÓREZ, P.; LEVIS, S.; RODAS, J.D. 2011. Genetic Evidence of Hantavirus Infections in Wild Rodents from Northwestern Colombia. Vector Borne Zoonotic Dis. 11:701-708.

MÁTTAR, S.; PARRA, M. 2004. Serologic Evidence of Hantavirus Infection in Humans, Colombia. Emerg Infect Dis. 10:2263-2264.

MILLS, J.N.; CHILDS, J.E.; KSIAZEK, T.G.; PETERS, C.J.; VELLECA, W.N. 1995. Methods for trapping and sampling small mammals for virology testing. U.S. Departament of Health \& Human Services.Public Health Service.Centers for Desease Control and Prevention. Atlanta, Georgia, EEUU. 
MILLS, J.N.; CHILDS, J.E. 1998. Ecologic studies of rodent reservoirs: their relevance for human health. Emerg Infect Dis. 4:529-538.

NAVARRETE, M.; SALDIAS, F.; MANCILLA, R.; ZAROR, L.; FERRES, M. 2000. Evidencia clínico epidemiológica de la existencia de síndrome pulmonar por hantavirus en Valdivia-Chile desde 1993. Rev Chil Infect. 17:230-240.

NICHOL, S.; SPIROPOULOU, C.; MORZUNOV, S.; ROLLIN, P.; KSIAZEK, T. 1993. Genetic identification of a hantavirus associated with an outbreak of acute respiratory illness. Science 262:914-917.

NOWAK, R.M. 1999. Walker's Mammals of the World. Vol. II, $6^{\text {th }}$ Edition. Johns Hopkins University Press, Baltimore, EEUU.

ORGANIZACIÓN PANAMERICANA DE LA SALUD (OPS). 2004. Número de casos de y defunciones por Síndrome Pulmonar por Hantavirus (SPH) (Región de las Américas, 1993-2004). [Internet]. Disponible en: http://www.paho.org/Spanish/AD/DPC/CD/hantavirus-1993-2004.htm.

Consultado 15-05-2012.

ORTIZ, J.C.; VENEGAS, W.; SANDOVAL, J.A.; CHANDIA, P.; TORRES, F. 2004. Hantavirus en roedores de la Octava Región de Chile. Rev Chil Hist Nat. 77:251-256.

PINI, N. 2004. Hantavirus Pulmonary Syndrome in Latin America. Curr Opin Infect Dis. 17:427-431.

PINI, N.; LEVIS, S.; CALDERÓN, G.; RAMIREZ, J.; BRAVO, D.; LOZANO, E.; RIPOLL, C.; ST-JEOR, S.; KSIAZEK, T.G.; BARQUEZ, R.M.; ENRIA, D. 2003. Hantavirus infection in humans and rodents, Northwestern Argentina. Emerg Infect Dis. 9:1070-1076.

PUERTA, H.; CANTILLO, C.; MILLS, J.; HJELLE, B.; SALAZAR-BRAVO, J.; MATTAR, S. 2006. Hantavirus del nuevo mundo. Ecología y epidemiología de un virus emergente en Latinoamérica. Medicina 66:343-356.

SCHMAL, J.C.; HJELLE, B. 1997. Hantaviruses: a global disease problem. Emerg Infect Dis. 3:95-104.

SCHOUNTZ, T.; CALISHER, C.H.; RICHENS, T.R.; RICH, A.A.; DOTY, J.B.; HUGHES, M.T.; BEATY, B.J. 2007. Rapid field immunoassay for detecting antibody to Sin Nombre virus in deer mice. Emerg Infect Dis. 13:1604-1607.

WILSON, M.L.; TESH, R.B.; FISH, D.; GERBER, M.A.; MAGNARELLI, L.A.; FEDER, H.M. JR.; SHAPIRO, E.D. 1995. Evidence of hantavirus infection in rodents and human beings from Connecticut and New York, USA. Lancet 345: 738. 